\title{
Quality control of gene expression: a stepwise assembly pathway for the surveillance complex that triggers nonsense-mediated mRNA decay
}

\author{
Isabelle Behm-Ansmant ${ }^{1}$ and Elisa Izaurralde $\mathrm{e}^{1,2,3}$ \\ ${ }^{1}$ European Molecular Biology Laboratory, D-69117 Heidelberg, Germany; ${ }^{2}$ Max-Planck-Institute for Developmental Biology, \\ D-72076 Tübingen, Germany
}

Eukaryotic cells have evolved elaborate mRNA quality control (surveillance) mechanisms to ensure that only fully processed and error-free mRNAs are translated. These quality control mechanisms operate in both the nucleus and the cytoplasm. For instance, in the nucleus, improperly processed mRNAs are degraded before they are transported to the cytoplasm (for review, see Fasken and Corbett 2005). In the cytoplasm, surveillance pathways assess the translatability of the mRNA and degrade any that have no translation termination codons (nonstop-mediated mRNA decay, NSD) or nonsense codons (nonsense-mediated mRNA decay, NMD), thereby preventing the accumulation of potentially toxic aberrant proteins (for review, see Conti and Izaurralde 2005; Fasken and Corbett 2005; Lejeune and Maquat 2005).

NMD is one of the best characterized mRNA surveillance pathways. Its importance as a protective surveillance mechanism is underscored by the fact that $~ 30 \%$ of inherited genetic disorders are caused by nonsense mutations or frameshifts, which generate nonsense codons (for review, see Frischmeyer and Dietz 1999; Holbrook et al. 2004). Transcripts derived from the mutant alleles are degraded by NMD, leading in general to a recessive mode of inheritance.

NMD not only rids the cell of mRNAs containing premature translation termination codons (PTCs or nonsense codons) as a result of mutations or errors in transcription or mRNA processing, but also regulates the expression of naturally occurring transcripts that represent $\sim 10 \%$ of the transcriptome in yeast, Drosophila, and human cells (Lelivelt and Culbertson 1999; He et al. 2003; Mendell et al. 2004; Rehwinkel et al. 2005). In this way, NMD plays a role in biological processes as diverse as telomere maintenance, transcription, cell proliferation, cell cycle, cellular transport and organization, and

${ }^{3}$ Corresponding author.

E-MAIL izaurralde@embl-heidelberg.de; FAX 49-6221-387-306.

Article and publication are at http://www.genesdev.org/cgi/doi/10.1101/ gad.1407606. metabolism. A deeper understanding of the mechanism underlying this pathway is therefore of critical importance.

NMD effectors forming a conserved protein interaction network

The RNA helicase UPF1 is a key effector of the NMD pathway. Deletion or silencing of the UPF1 gene results in the stabilization of PTC-containing mRNAs in all organisms in which the NMD pathway has been investigated (for review, see Conti and Izaurralde 2005; Lejeune and Maquat 2005). UPF1 activity is regulated by phosphorylation in multicellular organisms, and this requires interaction with two other conserved NMD effectors (UPF2, UPF3) and four additional proteins that, in contrast to UPF1-3, have no orthologs in yeast, namely, SMG1, SMG5, SMG6, and SMG7 (Pulak and Anderson 1993; Cali et al. 1999; Page et al. 1999). SMG1 is a phosphoinositide-3-kinase-related protein kinase required for UPF1 phosphorylation (Page et al. 1999; Denning et al. 2001; Pal et al. 2001; Yamashita et al. 2001; Grimson et al. 2004). SMG5, SMG6, and SMG7 can recognize phosphorylated UPF1 and are thought to trigger its dephosphorylation by recruiting protein phosphatase 2A (PP2A) (Anders et al. 2003; Chiu et al. 2003; Ohnishi et al. 2003).

The transient or stable interactions between NMD effectors lead to the formation of the so-called surveillance complex that assembles on mRNAs on which a premature translation termination event occurs. The surveillance complex, in turn, recruits RNA degradation enzymes and ultimately triggers the decay of the mRNA that served as platform for its own assembly (for review, see Conti and Izaurralde 2005; Lejeune and Maquat 2005). The role of the surveillance complex is therefore to couple a premature translation termination event to mRNA degradation. In agreement with this, NMD factors have genetic, functional, and/or physical interactions with both translation factors and mRNA decay enzymes. 
In this regard, the association of yeast UPF1, UPF2, and UPF3 with the eukaryotic translation termination factors eRF1 and eRF3 provides a link between NMD and translation termination (Czaplinski et al. 1998; Kobayashi et al. 2004). Moreover, multiple links between NMD factors and mRNA decay enzymes have been described in different organisms. These include the interaction of yeast UPF1 with the DCP1-DCP2 decapping complex, and the localization of human SMG7 to P-bodies, discrete cytoplasmic foci where mRNA degradation enzymes localize (for review; see Baker and Parker 2004; Conti and Izaurralde 2005).

\section{The mechanism of NMD: an overview}

In all eukaryotes a premature translation termination event leads to the assembly of the surveillance complex, yet the mechanisms by which a stop codon is defined as premature and the targeted mRNA is degraded differ across species. In mammals, PTC recognition relies on splicing: Stop codons are defined as premature if they are located $\sim 50$ nucleotides (nt) or more upstream of an exon-exon junction. In contrast, PTC recognition occurs independently of exon-exon boundaries in both Drosophila and Saccharomyces cerevisiae (for review, see Conti and Izaurralde 2005; Lejeune and Maquat 2005).

Degradation of the targeted mRNA involves many of the enzymes in the general pathway for mRNA turnover including the decapping DCP1-DCP2 complex, the $5^{\prime}$ to-3' exonuclease XRN1, deadenylases, and the 3 '-to-5' exonucleases of the exosome (for review, see Baker and Parker 2004). In yeast and human cells, the major decay pathway for NMD substrates is initiated by removal of the cap structure by the DCP1-DCP2 decapping complex. Following decapping, the body of the transcript is exposed to 5' -to-3' degradation by XRN1 (Cao and Parker 2003; Chen and Shyu 2003; Lejeune et al. 2003; Mitchell and Tollervey 2003; Couttet and Grange 2004). An alternative pathway, which also contributes to the decay of PTC-containing mRNAs, relies on the accelerated deadenylation and 3 '-to-5' degradation by the exosome /Cao and Parker 2003; Chen and Shyu 2003; Lejeune et al. 2003; Mitchell and Tollervey 2003). In Drosophila, degradation of nonsense transcripts is initiated by endonucleolytic cleavage near the PTC (Gatfield and Izaurralde 2004). The resulting $5^{\prime}$ decay intermediate is degraded by the exosome, while the 3' fragment is degraded by XRN1 (Gatfield and Izaurralde 2004). Thus, in this case, the mRNA fragments are degraded from the newly generated ends without being decapped or deadenylated, suggesting that, in contrast to yeast and mammals, the decapping enzymes and deadenylases may not interact with NMD effectors in Drosophila.

As a consequence of the differences in the mechanisms of PTC recognition and mRNA decay described above, the full set of proteins required for NMD must differ across species. A striking example of this is provided in mammals, where the dependence on exon-exon boundaries for PTC recognition concurs with the requirements of components of the exon junction complex
(EJC) for NMD in these organisms (for review, see Lejeune and Maquat 2005). Indeed, the positions of exon-exon boundaries are communicated to translating ribosomes by the EJC, a multiprotein assembly deposited by the spliceosome 20-24 nt upstream of exon-exon junctions (Le Hir et al. 2000). Consistent with this, the EJC components Y14, MAGOH, eIF4AIII, Barentsz, and RNPS1, function in NMD (Lykke-Andersen et al. 2001; Fribourg et al. 2003; Gehring et al. 2003, 2005; Ferraiuolo et al. 2004; Palacios et al. 2004; Shibuya et al. 2004). These proteins interact with mammalian UPF3, which is also loaded onto mRNAs during splicing and represents a genuine EJC component (Kim et al. 2001; Le Hir et al. 2001).

As mentioned above, in Drosophila as well as in yeast, PTC recognition occurs independently of exon-exon junctions, and although orthologs of mammalian EJC proteins (i.e., Y14, MAGOH, eIF4AIII, Barentsz, and RNPS1) are conserved in Drosophila, they are not essential for NMD (Gatfield et al. 2003), suggesting that EJC components have been co-opted by the NMD machinery after the divergence of vertebrates and invertebrates. None of these proteins has orthologs in S. cerevisiae.

\section{A trimeric UPF1:UPF2:UPF3 complex forms the conserved core of the NMD machinery}

Based on several lines of evidence, a model for NMD in mammals has emerged in which UPF3 is loaded onto mRNAs during splicing, while UPF2 is thought to join the complex in the cytoplasm after export. During the first round of translation, UPF2, UPF3, and the additional EJC components are displaced by the ribosomes as they traverse the mRNA. If translating ribosomes were to encounter a stop codon upstream of an EJC, this would lead to the incomplete removal of UPF2 and UPF3 proteins from downstream mRNA sequences and to the recruitment of UPF1, probably via interactions with the eRF1-eRF3 complex (see below). The recruitment of UPF1 creates an opportunity for the assembly of the surveillance complex consisting of UPF1, UPF2, and UPF3, which triggers UPF1 phosphorylation and ultimately targets the mRNA for rapid degradation.

A key step in this model is the formation of the trimeric UPF1:UPF2:UPF3 complex, which represents the conserved core of the NMD machinery and is thought to assemble in all organisms independently of the mechanism by which PTCs are defined (for review, see Conti and Izaurralde 2005; Lejeune and Maquat 2005). Formation of the trimeric complex is supported by genetic, biochemical, and structural evidence. UPF2 interacts directly with UPF3 (Lykke-Andersen et al. 2000; Serin et al. 2001; Kadlec et al. 2004 and references therein). This interaction has been visualized at the atomic level (Kadlec et al. 2004). UPF2 consists of three MIF4G (middle portion of eIF4G) domains, while UPF3 is characterized by a canonical RNP-type RNA-binding domain (RBD) (Kadlec et al. 2004). The structure of the complex between the interacting domains of human UPF2 and UPF3b (two UPF3 paralogs exist in humans: a and b) 
shows that this interaction involves the third MIF4G domain of UPF2 and the UPF3 RBD (Kadlec et al. 2004). The conservation of the interactions and surfaces indicates that the UPF2:UPF3 complex has similar features in all organisms. UPF2 also interacts with UPF1. The UPF1-binding site has been mapped to the $\mathrm{N}$ - and Cterminal regions of UPF2 (He et al. 1996). In the UPF2:UPF3 complex, these regions are likely to be accessible for interaction with UPF1, allowing the assembly of the trimeric core of the surveillance complex.

Genetic studies both in S. cerevisiae and Caenorhabditis elegans and gene knockdowns in Drosophila have shown that UPF1, UPF2, and UPF3 act as obligate partners in the NMD pathway; this means that the absence of any of these proteins leads to the stabilization of PTCcontaining mRNAs, and thus the inhibition of the NMD pathway (Pulak and Anderson 1993; Lelivelt and Culbertson 1999; Gatfield et al. 2003). These genetic and knockdown studies focused on a small number of reporters and the possibility that some NMD targets bypass the requirement for the formation of the trimeric complex could not be ruled out. More recently, the analysis of whole transcriptomes by gene expression profiles in cells lacking individual NMD factors also revealed that these proteins regulate the expression of a common set of transcripts in both yeast and Drosophila cells. Indeed, yeast strains lacking UPF1, UPF2, or UPF3 exhibit similar expression profiles (He et al. 2003). Similarly, depletion of UPF1, UPF2, UPF3, SMG1, SMG5, or SMG6 from Drosophila cells results in correlated changes in gene expression (Rehwinkel et al. 2005). In particular, cells depleted of UPF1 or UPF2 exhibit strikingly similar expression profiles (Rehwinkel et al. 2005), excluding the possibility of a UPF2-independent NMD pathway in this organism (see below).

\section{Alternative NMD pathways in human cells}

Gehring et al. (2005) provide evidence that, contrary to expectation, two alternative complexes can trigger NMD in human cells. Both complexes contain UPF1, but differ in their requirement for UPF2. Gehring et al. (2005) used a tethering assay to investigate the requirement for different NMD factors and EJC components to trigger mRNA decay. This assay involves the expression of NMD effectors or EJC components fused to the RNAbinding motif of the $N$ protein of bacteriophage $\lambda / \lambda N$ protein) or to the coat protein of MS2 bacteriophage (MS2 coat protein). These proteins will bind with high affinity to specific RNA sequences that are inserted in the 3' UTR of a reporter mRNA (Fig. 1). When NMD factors or EJC components are artificially tethered to the reporter in this way and are positioned at least $50 \mathrm{nt}$ downstream of the natural stop codon, the natural stop is perceived as premature and the mRNA is degraded. This has been shown for the NMD effectors, UPF1, UPF2, UPF3a/b, as well as for the EJC components, Y14, MAGOH, Barentsz, eIF4AIII, and RNPS1 (Lykke-Andersen et al. 2000, 2001; Fribourg et al. 2003; Gehring et al. 2003, 2005; Ferraiuolo et al. 2004; Palacios et al. 2004; Shibuya et al. 2004).

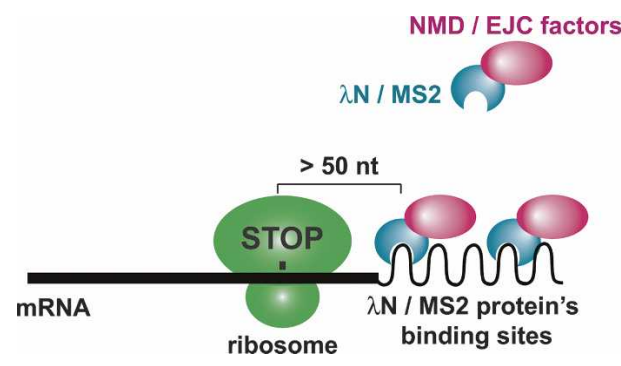

Figure 1. The tethering assay. Proteins of interest are fused to the MS2 coat protein or to the RNA-binding motif of the $\lambda \mathrm{N}$ protein. These proteins bind with high affinity to their respective binding sites inserted in the 3' UTR of the reporter. The artificial binding of NMD factors or EJC components to the reporter elicits its degradation, provided that the tethering sites are at least $50 \mathrm{nt}$ downstream of the natural stop codon.

In tethering assays, NMD can be triggered by the assembly of a complex consisting of UPF1, UPF3b, Y14, MAGOH, Barentsz, and eIF4AIII (Gehring et al. 2005). This NMD pathway is insensitive to the depletion of UPF2, but is inhibited by depletion of Barentsz or eIF4AIII. In an alternative pathway, NMD is triggered by the association of UPF1 with RNPS1 and UPF2, and is insensitive to the depletion of Barentsz or eIF4AIII (Gehring et al. 2005). These observations suggest that EJC components have not only been co-opted by the NMD machinery during evolution, but some of these components may have acquired a role similar to that of UPF2. In particular, the tetrameric complex of Y14, MAGOH, Barentsz, and eIF4AIII appears to be able to substitute for UPF2 (Gehring et al. 2005).

In agreement with the existence of a UPF2-independent NMD pathway, earlier studies by Gehring et al. (2003) showed that the requirement for UPF2-UPF3b interaction is bypassed when UPF3b is tethered to a reporter transcript. More precisely, a UPF3b mutant lacking the RBD elicits NMD in tethering assays. Together, the results reported by Gehring et al. $(2003,2005)$ suggest that for a subset of targets, NMD is active either in the absence of UPF2, or when the protein levels are strongly reduced.

The lack of genome-wide information on genes regulated by key components of the NMD machinery in human cells (i.e., UPF1, UPF2, UPF3a,b), left open the question of whether NMD targets that are UPF2-independent exist. Gehring et al. (2005) provide evidence for the existence of this class of targets. Analysis of gene expression profiles in cells depleted of UPF1, UPF2, RNPS1, or Barentsz allowed those authors to identify transcripts regulated by UPF1 and Barentsz, but insensitive to the depletion of UPF2, and transcripts regulated by UPF1, UPF2, and RNPS1, but insensitive to Barentsz depletion. These results strongly support the existence in human cells of alternative NMD pathways that converge at a common requirement for UPF1. Gehring et al. (2005) also identified targets regulated by both UPF2 and Barentsz, suggesting that formation of the trimeric UPF1:UPF2:UPF3 complex is required for the degradation of these targets. 


\section{A stepwise assembly pathway for the surveillance complex}

Prior to its interaction with UPF3 and/or UPF2 bound to downstream EJC components, UPF1 is thought to be recruited to PTC-containing mRNAs via interactions with the release factors (eRF1 and eRF3) when premature translation termination occurs. However, until now there was no direct biochemical evidence to support this model in higher eukaryotes. Moreover, although earlier studies had shown that phosphorylation of UPF1 by SMG1 is essential for NMD (Page et al. 1999; Denning et al. 2001; Pal et al. 2001; Yamashita et al. 2001; Anders et al. 2003; Ohnishi et al. 2003; Grimson et al. 2004), the mechanism by which UPF2 and UPF3 contributed to this process, as well as the link between UPF1 phosphorylation and premature translation termination, were not defined.

In a recent report in Genes \& Development, Kashima et al. (2006) aim to investigate the mechanism of UPF1 phosphorylation. They begin by performing immunoprecipitations from cytoplasmic human cell extracts using anti-SMG1 antibodies. Their exciting new finding is the observation that SMG1 (endogenous or ectopically expressed) coimmunoprecipitates with UPF1, UPF2, UPF3a/b, Y14, MAGOH, eIF4AIII, and SMG7 even in the presence of RNases, suggesting that these interactions are not mediated by RNA. The nuclear cap-binding protein CBP20, and the poly(A)-binding protein PABP1 are also detected in this complex, but their association is mediated by RNA and requires Y14. In contrast, the cytoplasmic cap-binding protein eIF4E is not coimmunoprecipitated. These data indicate that Y14 bridges the association of SMG1 with spliced mRNAs that are still bound to the nuclear cap-binding complex (CBC).

To identify the domains of SMG1 involved in these interactions, the authors performed immunoprecipitations with $\mathrm{N}$ - and C-terminal fragments of SMG1. The $\mathrm{N}$-terminal domain of SMG1 (amino acids 1-2223) coimmunoprecipitates with UPF1, UPF3a, and SMG7, but fails to precipitate EJC components. The C-terminal domain of SMG1 (amino acids 2068-3657) associates with UPF1, UPF2, UPF3b, Y14, and MAGOH, suggesting that this domain mediates the interaction with EJC components. To investigate which of the SMG1 partners is more likely to interact directly with SMG1, they performed pull-down experiments with purified recombinant $\mathrm{N}$ - and C-terminal domains of SMG1 and in vitro translated proteins. The purified $\mathrm{N}$-terminal domain of SMG1 interacts with UPF1, while the C-terminal domain interacts with UPF2, but not with UPF3a/b or other EJC components. Thus, the association of SMG1 or its C-terminal domain with UPF3b and EJC components observed in cell lysates is most likely mediated by UPF2. In agreement with this, in cells depleted of UPF2, the amount of Y14 coimmunoprecipitating with SMG1 is strongly reduced (Kashima et al. 2006). Additional evidence for the formation of a complex between SMG1, UPF1, UPF2, UPF3b, and Y14 is obtained in coimmunoprecipitation assays using anti-Y14 or anti-UPF2 anti- bodies. In both cases a complex consisting minimally of UPF2, UPF3b, and Y14 is observed.

Since there is no evidence for a direct interaction between UPF2 and the EJC, Kashima et al. (2006) reasoned that UPF3b bridges this interaction. In other words, UPF2:UPF3 heterodimerization is required for the association of EJC components with SMG1. To obtain additional evidence for the requirement of UPF2:UPF3b heterodimerization, Kashima et al. (2006) made use of two previously described UPF2 mutants that disrupt the interaction with UPF3b, in particular, a mutant carrying a deletion of the third MIF4G domain of UPF2 (amino acids 711-928) (Serin et al. 2001) and a mutant in which a single glutamic acid (E858) in the third MIF4G domain of UPF2 is replaced by an arginine (Kadlec et al. 2004). As expected, these mutants failed to coimmunoprecipitate UPF3b, but also Y14, providing further evidence to support the proposal that UPF2 interaction with Y14 is mediated by UPF3b. More importantly, overexpression of UPF2 mutants inhibits the association of Y14 with SMG1 in a dominant-negative manner, while overexpression of wild-type UPF2 has no effect. Remarkably, these UPF2 mutants inhibit NMD when overexpressed. Indeed, the half-life of a PTC-carrying $\beta$-globin mRNA reporter increased in cells expressing the UPF2 mutants, but not wild-type UPF2 protein. The half-life of the corresponding mRNA reporter lacking a PTC was not affected by the overexpression of any of these proteins.

What could be the role of the association of SMG1 with UPF1, UPF2, UPF3, and EJC components? One obvious possibility is that formation of this complex is required to trigger UPF1 phosphorylation. Using a specific antibody raised to phosphorylated UPF1, Kashima et al. (2006) show that depletion of UPF2, UPF3b, or Y14 strongly reduces the amount of the phosphorylated form of UPF1, indicating that UPF1 is, indeed, phosphorylated as a consequence of its association with UPF2, UPF3b, Y14, and additional EJC components. One implication of these results is that UPF2, UPF3b, and Y14 are all required to trigger UPF1 phosphorylation and that neither UPF3b nor Y14 can substitute for UPF2. In agreement with this, the UPF2 mutants that no longer interact with UPF3b and prevent the association of SMG1 with Y14 also decrease the phosphorylation of UPF1, providing an explanation for their inhibitory effect in NMD.

A striking result of the work of Kashima et al. (2006) is the observation that in cells depleted of UPF2 or Y14, UPF1 is not phosphorylated, but its interaction with SMG1 is stimulated. The authors reasoned that SMG1 could form a complex with UPF1 in the absence of UPF2 and EJC components, and that this complex could represent an early step in the pathway, before its association with EJC components on the mRNA. They speculate that if this were the case, the release factors may be part of this complex. They found that the association of eRF1 and eRF3 with SMG1 and UPF1 is stimulated in Y14- or UPF2-depleted cells. Similarly, a UPF1 mutant that does not interact with UPF2 (UPF1-C126S) shows increased interaction with SMG1, eRF1, and eRF3. This mutant is not phosphorylated and inhibits NMD when overex- 
pressed. Finally, overexpression of a kinase inactive mutant of SMG1, which inhibits UPF1 phosphorylation in a dominant-negative manner, also stimulates the association of UPF1 with eRF1 and eRF3. These results suggest that phosphorylation of UPF1 induces the dissociation of eRF1 and eRF3.

Together, the results of Kashima et al. (2006) reveal the existence of two novel protein complexes that represent consecutive steps in the NMD pathway (Fig. 2). One complex (the SURF complex) consists of SMG1, UPF1, and the release factors eRF1 and eRF3, and a second complex consists minimally of SMG1, UPF1 UPF2, UPF3b, Y14, MAGOH, and eIF4AIII (Fig. 2). Although Kashima et al. (2006) did not investigate the presence of Barentsz in this complex, it is likely that it is also present, as this protein forms a stable complex with Y14, MAGOH, and eIF4AIII (Ferraiuolo et al. 2004; Palacios et al. 2004; Shibuya et al. 2004; Gehring et al. 2005).

The SURF complex appears to be transient as it can be detected in cells in which UPF1 phosphorylation is inhibited. This can be done with three different approaches: by depleting UPF2 or Y14, by overexpressing UPF2 mutants that do not interact with UPF3b, or by overexpressing an inactive form of SMG1. In all three conditions, UPF1 phosphorylation is strongly reduced and the association of eRF1 and eRF3 with UPF1 and SMG1 is stimulated. Thus, SURF accumulates when the next step in the NMD pathway, the phosphorylation of UPF1, is inhibited.

What is the link between the two complexes described by Kashima et al. (2006)? They propose that SURF assembles on ribosomes stalled at a PTC (Fig. 2). The interaction of SURF with UPF2, UPF3b, Y14, and other EJC components bound to a downstream exon-exon boundary leads to the formation of a transient complex, the SURF:EJC complex, termed decay-inducing complex (DECID). DECID triggers UPF1 phosphorylation and the dissociation of eRF1 and eRF3, and gives birth to the second complex that they describe. It should be noted that DECID is inferred to exist by the authors but not isolated as a biochemical entity in their study. Nevertheless, DECID represents the most plausible transition between SURF and the SMG1-UPF1-UPF2-UPF3b-EJC complex. The RNA-dependent association of CBP20 and PABP1 with these two complexes suggests that their assembly occurs on spliced mRNAs that are still bound to the nuclear CBC. The observation that the cytoplasmic cap-binding protein, eIF4E, is not present in these complexes is not an unexpected result as eIF4E-bound mRNAs are actively translated and EJC components are thought to be displaced from the mRNA by translating ribosomes (Dostie and Dreyfuss 2002).

\section{UPF2-independent pathway: a minor pathway for mammalian NMD?}

The existence of a complex consisting of SMG1, UPF1, UPF2, UPF3b, Y14, and additional EJC components required to trigger UPF1 phosphorylation and NMD, as shown by Kashima et al. (2006), is consistent with re-
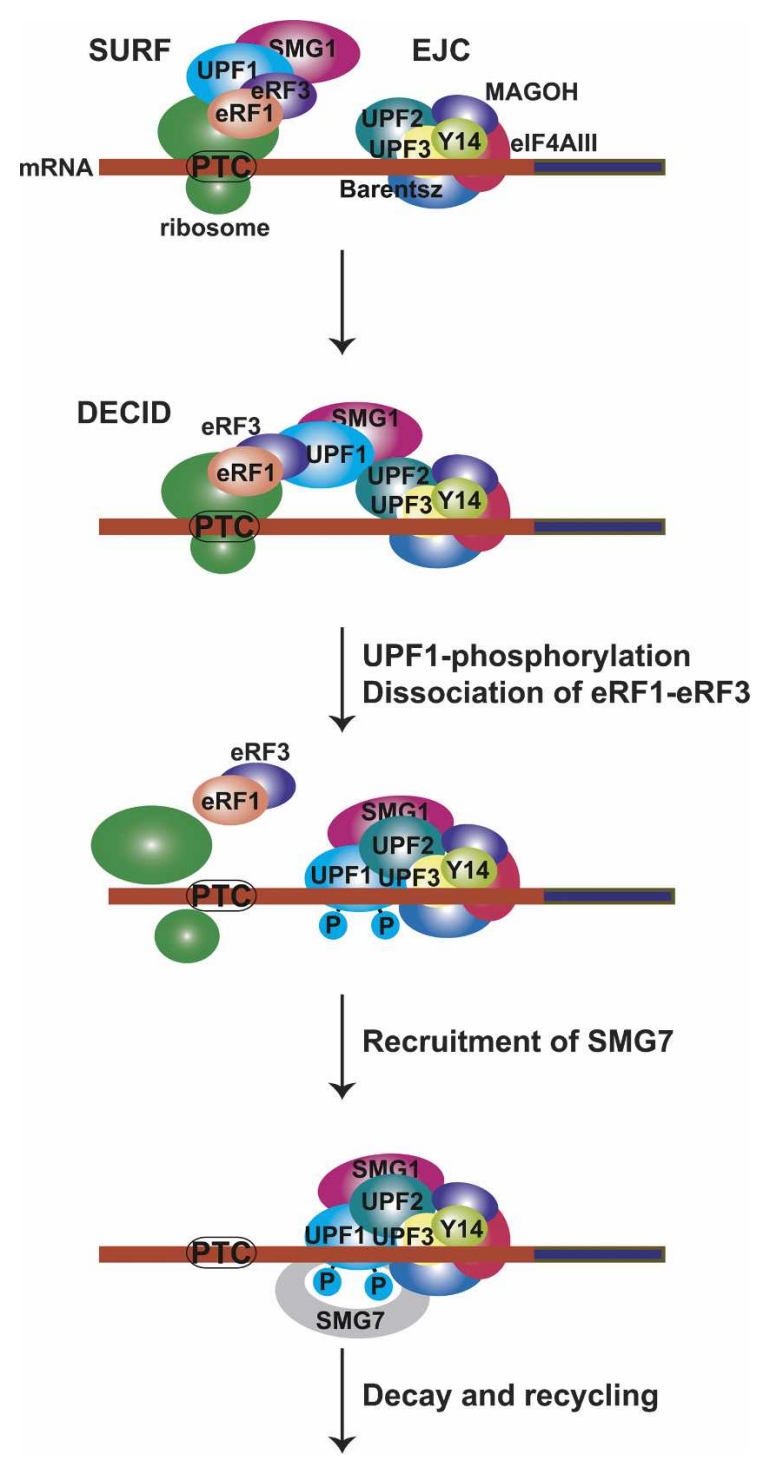

Figure 2. A model for mammalian NMD. A premature translation termination event leads to the assembly of the SURF complex consisting of SMG1, UPF1, eRF1, and eRF3. SURF interacts with UPF2, UPF3, and additional EJC proteins bound to a downstream exon-exon boundary. This interaction results in the formation of the DECID complex that triggers UPF1 phosphorylation and the dissociation of eRF1 and eRF3. Phosphorylated UPF1 recruits SMG7 most likely in association with SMG5. The interaction of SMG7 (and probably additional proteins) with decay enzymes targets the bound mRNA for degradation (not shown). SMG7 also recruits PP2A, resulting in UPF1 dephosphorylation and dissociation from SMG7 (not shown). This may enable the recycling of these proteins for a new round of NMD. (Red and blue boxes) Exons; (PTC) premature translation termination codon.

sults in other organisms showing that UPF1 phosphorylation and NMD require the formation of a trimeric UPF1:UPF2:UPF3 complex. These results are, at first glance, more difficult to reconcile with the UPF2-independent pathway proposed by Gehring et al. (2005). If the UPF2-independent pathway represents a major pathway, 
one would expect UPF1 phosphorylation not to be affected by UPF2 depletion, as in the absence of UPF2, NMD should still be functional.

How can the differences between these studies be explained? At least a partial answer to this question could depend on the extent of the contribution of the alternative pathways to global NMD, that is, the UPF2-independent pathway could represent a minor pathway-a possibility not excluded by Gehring et al. (2005). Another possible answer may depend on the nature of the assays used in these studies, that is, tethering assay versus PTC-containing mRNA reporters, raising the question of how well the tethering assay reflects genuine NMD. It has been reported that human UPF1 can be recruited to the 3' UTR of specific transcripts via interactions with Staufen-1 or the histone-stem-loop-binding protein and elicits mRNA decay by a mechanism distinct from but related to NMD (Kaygun and Marzluf 2005; Kim et al. 2005). In particular, these decay pathways are independent of UPF2. By analogy, other NMD factors or EJC components may have acquired roles in post-transcriptional processes distinct from NMD. In this case, the tethering assay may not always reflect the NMD pathway. The observation that a subset of endogenous transcripts is regulated by UPF1, but is insensitive to UPF2 depletion, not only provides evidence for a UPF2-independent NMD pathway, but may also reflect additional roles of UPF1 in mRNA turnover that are not related to NMD. Most importantly, eukaryotic 3' UTRs are sites for a wide variety of post-transcriptional regulatory elements, and tethering proteins to the 3' UTRs of transcripts may have unpredictable effects on translation and/or mRNA turnover.

On the other hand, the stabilization of PTC-containing mRNAs in cells depleted of EJC components may not reflect a direct role of these components in the NMD pathway, but secondary effects of the depletion on EJC assembly and/or translation efficiency.

In short, the studies of Gehring et al. (2005) and Kashima et al. (2006) provide important new insights into the NMD pathway but clearly indicate that our understanding of this pathway is incomplete and more studies are needed to clarify whether alternative NMD pathways have, indeed, evolved in higher eukaryotes.

\section{Role of UPF1 phosphorylation}

The phosphorylation of UPF1 is thought to recruit SMG5, SMG6, and SMG7, three similar but not functionally redundant proteins, which are thought to trigger UPF1 dephosphorylation by recruiting PP2A. This model was first suggested by genetic studies in C. elegans that identified SMG5, SMG6, and SMG7 as proteins required for UPF1 dephosphorylation (Page et al. 1999; Denning et al. 2001; Pal et al. 2001). Moreover, biochemical studies in both C. elegans and human cells have shown that SMG5 and SMG7 interact with each other and are part of a larger complex containing PP2A and phosphorylated UPF1 (Anders et al. 2003; Ohnishi et al. 2003). Similarly, SMG6 is part of a protein complex containing PP2A and phosphorylated UPF1 (Chiu et al. 2003). Kashima et al. (2006) also detected SMG7 in the SMG1-UPF1-UPF2EJC complex, and in a complex formed with the N-terminal domain of SMG1 and UPF1, indicating that additional interactions may contribute to the recruitment of SMG5-7.

The crystal structure of the $\mathrm{N}$-terminal domain of SMG7 has revealed the molecular basis for the interaction of SMG7 with the phosphorylated form of UPF1. The N-terminal domain of SMG7 resembles that of 143-3, a phosphoserine-binding protein involved in signal transduction pathways (Fukuhara et al. 2005). The similarity between SMG7 and 14-3-3 is not limited to their overall fold, but extends to most of the residues that line the 14-3-3 phosphoserine-binding pocket. Indeed, SMG7 binds UPF1 in a phosphorylation-dependent manner, and mutation of residues in its 14-3-3-like phosphoserinebinding site impairs UPF1 binding (Fukuhara et al. 2005). Sequence alignments suggest that SMG5 and SMG6 also contain a 14-3-3-like domain with a conserved binding site for phosphoserine residues most likely in UPF1 (Fukuhara et al. 2005).

In addition to its $\mathrm{N}$-terminal 14-3-3-like domain, SMG7 has a C-terminal domain that targets bound mRNAs for decay (Unterholzner and Izaurralde 2004). When overexpressed, SMG7 accumulates in cytoplasmic foci corresponding to endogenous P-bodies and causes the accumulation of SMG5 or UPF1 in P-bodies. This suggests that SMG7 is one of the components of the surveillance complex that couples changes in the phosphorylation state of UPF1 with mRNA degradation.

\section{An extended model for mammalian NMD}

The findings of Kashima et al. (2006) provide mechanistic insights into the assembly of the surveillance complex and into the steps that lead to the phosphorylation of UPF1 (Fig. 2). Accordingly, a premature translation termination event leads to the assembly of the SURF complex on the aberrant mRNA. SURF interacts with UPF2, UPF3, and EJC components bound to a downstream exon boundary, and this interaction triggers UPF1 phosphorylation. Phosphorylated UPF1 interacts with SMG7 (probably in a complex with SMG5 and PP2A). SMG7 would then target the PTC-containing transcript for decay by a mechanism yet to be determined. The association of SMG7, SMG5, and PP2A would also trigger the dephosphorylation of UPF1, and this event might be involved in recycling of the NMD factors for another round of NMD. This model does not exclude the possibility that the requirement for UPF2 can be bypassed by a specific constellation of EJC proteins bound to a subset of targets, as indicated by the work of Gehring et al. (2005).

The model emerging from the studies of Kashima et al. (2006) ties together years of accumulated genetic, biochemical, and structural data, and although additional studies are required for a molecular understanding of the mechanism underlying NMD, this model makes testable 
predictions that will certainly give way to future research.

\section{Acknowledgments}

We gratefully acknowledge Niels Gehring, Matthias Hentze, and David Thomas for critical reading of the manuscript. The authors are supported by the European Molecular Biology Organization (EMBO) and the Human Frontier Science Program Organization (HFSPO).

\section{References}

Anders, K.R., Grimson, A., and Anderson, P. 2003. SMG-5, required for $C$. elegans nonsense-mediated mRNA decay, associates with SMG-2 and protein phosphatase 2A. EMBO J. 22: 641-650.

Baker, K.E. and Parker, R. 2004. Nonsense-mediated mRNA decay: Terminating erroneous gene expression. Curr. Opin. Cell Biol. 16: 293-299.

Cali, B.M., Kuchma, S.L., Latham, J., and Anderson, P. 1999. smg-7 is required for mRNA surveillance in Caenorhabditis elegans. Genetics 151: 605-616.

Cao, D. and Parker, R. 2003. Computational modeling and experimental analysis of nonsense-mediated decay in yeast. Cell 113: 533-545.

Chen, C.Y. and Shyu, A.B. 2003. Rapid deadenylation triggered by a nonsense codon precedes decay of the RNA body in a mammalian cytoplasmic nonsense-mediated decay pathway. Mol. Cell. Biol. 23: 4805-4813.

Chiu, S.Y., Serin, G., Ohara, O., and Maquat, L.E. 2003. Characterization of human Smg5/7a: A protein with similarities to Caenorhabditis elegans SMG5 and SMG7 that functions in the dephosphorylation of Upf1. RNA 9: 77-87.

Conti, E. and Izaurralde, E. 2005. Nonsense-mediated mRNA decay: Molecular insights and mechanistic variations across species. Curr. Opin. Cell Biol. 17: 316-325.

Couttet, P. and Grange, T. 2004. Premature termination codons enhance mRNA decapping in human cells. Nucleic Acids Res. 32: 488-494.

Czaplinski, K., Ruiz-Echevarria, M.J., Paushkin, S.V., Han, X., Weng, Y., Perlick, H.A., Dietz, H.C., Ter-Avanesyan, M.D., and Peltz, S.W. 1998. The surveillance complex interacts with the translation release factors to enhance termination and degrade aberrant mRNAs. Genes \& Dev. 12: 1665-1677.

Denning, G., Jamieson, L., Maquat, L.E., Thompson, E.A., and Fields, A.P. 2001. Cloning of a novel phosphatidylinositol kinase-related kinase: Characterization of the human SMG-1 RNA surveillance protein. I. Biol. Chem. 276: 22709-22714.

Dostie, J. and Dreyfuss, G. 2002. Translation is required to remove Y14 from mRNAs in the cytoplasm. Curr. Biol. 12: 1060-1067.

Fasken, M.B. and Corbett, A.H. 2005. Process or perish: Quality control in mRNA biogenesis. Nat. Struct. Mol. Biol. 12: 482488.

Ferraiuolo, M.A., Lee, C.S., Ler, L.W., Hsu, J.L., Costa-Mattioli, M., Luo, M.J., Reed, R., and Sonenberg, N. 2004. A nuclear translation-like factor eIF4AIII is recruited to the mRNA during splicing and functions in nonsense-mediated decay. Proc. Nat1. Acad. Sci. 101: 4118-4123.

Fribourg, S., Gatfield, D., Izaurralde, E., and Conti, E. 2003. A novel mode of RBD-protein recognition in the Y14-Mago complex. Nat. Struct. Biol. 10: 433-439.
Frischmeyer, P.A. and Dietz, H.C. 1999. Nonsense-mediated mRNA decay in health and disease. Hum. Mol. Genet. 8: 1893-1900.

Fukuhara, N., Ebert, J., Unterholzner, L., Lindner, D., Izaurralde, E., and Conti, E. 2005. SMG7 is a 14-3-3-like adaptor in the nonsense-mediated mRNA decay pathway. Mol. Cell 18: $537-547$.

Gatfield, D. and Izaurralde, E. 2004. Nonsense-mediated messenger RNA decay is initiated by endonucleolytic cleavage in Drosophila. Nature 429: 575-578.

Gatfield, D., Unterholzner, L., Ciccarelli, F.D., Bork, P., and Izaurralde, E. 2003. Nonsense-mediated mRNA decay in Drosophila: At the intersection of the yeast and mammalian pathways. EMBO I. 22: 3960-3970.

Gehring, N.H., Neu-Yilik, G., Schell, T., Hentze, M.W., and Kulozik, A.E. 2003. Y14 and hUpf3b form an NMD-activating complex. Mol. Cell 11: 939-949.

Gehring, N.H., Kunz, J.B., Neu-Yilik, G., Breit, S., Viegas, M.H., Hentze, M.W., and Kulozik, A.E. 2005. Exon-junction complex components specify distinct routes of nonsense-mediated mRNA decay with differential cofactor requirements. Mol. Cell 20: 65-75.

Grimson, A., O'Connor, S., Newman, C.L., and Anderson, P. 2004. SMG-1 is a phosphatidylinositol kinase-related protein kinase required for nonsense-mediated mRNA decay in Caenorhabditis elegans. Mol. Cell. Biol. 17: 7483-7490.

He, F., Brown, A.H., and Jacobson, A. 1996. Interaction between $\mathrm{Nmd} 2 \mathrm{p}$ and Upf1p is required for activity but not for dominant-negative inhibition of the nonsense-mediated mRNA decay pathway in yeast. RNA 2: 153-170.

He, F., Li, X., Spatrick, P., Casillo, R., Dong, S., and Jacobson, A. 2003. Genome-wide analysis of mRNAs regulated by the nonsense-mediated and $5^{\prime}$ to $3^{\prime}$ mRNA decay pathways in yeast. Mol. Cell 12: 1439-1452.

Holbrook, J.A., Neu-Yilik, G., Hentze, M.W., and Kulozik, A.E. 2004. Nonsense-mediated decay approaches the clinic. Nat. Genet. 36: 801-808.

Kadlec, J., Izaurralde, E., and Cusack, S. 2004. The structural basis for the interaction between nonsense-mediated mRNA decay factors UPF2 and UPF3. Nat. Struct. Mol. Biol. 11: 330-337.

Kashima, I., Yamashita, A., Izumi, N., Kataoka, N., Morishita, R., Hoshino, S., Ohno, M., Dreyfuss, G., and Ohno, S. 2006. Binding of a novel SMG-1-Upf1-eRF1-eRF3 complex (SURF) to the exon junction complex triggers Upf1 phosphorylation and nonsense-mediated mRNA decay. Genes \& Dev. 20: 355-367.

Kaygun, H. and Marzluf, W.F. 2005. Regulated degradation of replication-dependent histone mRNAs requires both ATR and Upf1. Nat. Struct. Mol. Biol. 12: 794-800.

Kim, V.N., Kataoka, N., and Dreyfuss, G. 2001. Role of the nonsense-mediated decay factor hUpf3 in the splicing-dependent exon-exon junction complex. Science 293: 1832 1836.

Kim, Y.K., Furic, L., Desgroseillers, L., and Maquat, L.E. 2005. Mammalian Staufen1 recruits Upf1 to specific mRNA 3'UTRs so as to elicit mRNA decay. Cell 120: 195-208.

Kobayashi, T., Funakoshi, Y., Hoshino, S., and Katada, T. 2004. The GTP-binding release factor eRF3 as a key mediator coupling translation termination to mRNA decay. J. Biol. Chem. 279: 45693-45700.

Le Hir, H., Izaurralde, E., Maquat, L.E., and Moore, M.J. 2000. The spliceosome deposits multiple proteins 20-24 nucleotides upstream of mRNA exon-exon junctions. EMBO J. 19: 6860-6869.

Le Hir, H., Gatfield, D., Izaurralde, E., and Moore, M.J. 2001. 
The exon-exon junction complex provides a binding platform for factors involved in mRNA export and nonsensemediated mRNA decay. EMBO J. 20: 4987-4997.

Lejeune, F. and Maquat, L.E. 2005. Mechanistic links between nonsense-mediated mRNA decay and pre-mRNA splicing in mammalian cells. Curr. Opin. Cell Biol. 17: 309-315.

Lejeune, F., Li, X., and Maquat, L.E. 2003. Nonsense-mediated mRNA decay in mammalian cells involves decapping, deadenylating, and exonucleolytic activities. Mol. Cell 12: 675687.

Lelivelt, M.J. and Culbertson, M.R. 1999. Yeast Upf proteins required for RNA surveillance affect global expression of the yeast transcriptome. Mol. Cell. Biol. 19: 6710-6719.

Lykke-Andersen, J., Shu, M.D., and Steitz, J.A. 2000. Human Upf proteins target an mRNA for nonsense-mediated decay when bound downstream of a termination codon. Cell 103: $1121-1131$.

- 2001. Communication of the position of exon-exon junctions to the mRNA surveillance machinery by the protein RNPS1. Science 293: 1836-1839.

Mendell, J.T., Sharifi, N.A., Meyers, J.L., Martinez-Murillo, F., and Dietz, H.C. 2004. Nonsense surveillance regulates expression of diverse classes of mammalian transcripts and mutes genomic noise. Nat. Genet. 36: 1073-1078.

Mitchell, P. and Tollervey, D. 2003. An NMD pathway in yeast involving accelerated deadenylation and exosome-mediated 3' $\rightarrow$ 5' degradation. Mol. Cell 11: 1405-1413.

Ohnishi, T., Yamashita, A., Kashima, I., Schell, T., Anders, K.R., Grimson, A., Hachiya, T., Hentze, M.W., Anderson, P., and Ohno, S. 2003. Phosphorylation of hUPF1 induces formation of mRNA surveillance complexes containing hSMG-5 and hSMG-7. Mol. Cell 12: 1187-1200.

Page, M.F., Carr, B., Anders, K.R., Grimson, A., and Anderson, P. 1999. SMG-2 is a phosphorylated protein required for mRNA surveillance in Caenorhabditis elegans and related to Upflp of yeast. Mol. Cell. Biol. 19: 5943-5951.

Pal, M., Ishigaki, Y., Nagy, E., and Maquat, L.E. 2001. Evidence that phosphorylation of human Upfl protein varies with intracellular location and is mediated by a wortmannin-sensitive and rapamycin-sensitive PI 3-kinase-related kinase signaling pathway. RNA 7: 5-15.

Palacios, I.M., Gatfield, D., St Johnston, D., and Izaurralde, E. 2004. An eIF4AIII-containing complex required for mRNA localization and nonsense-mediated mRNA decay. Nature 427: 753-757.

Pulak, R. and Anderson, P. 1993. mRNA surveillance by the Caenorhabditis elegans smg genes. Genes \& Dev. 7: 18851897.

Rehwinkel, J., Letunic, I., Raes, J., Bork, P., and Izaurralde, E. 2005. Nonsense-mediated mRNA decay factors act in concert to regulate common mRNA targets. RNA 11: $1530-1544$.

Serin, G., Gersappe, A., Black, J.D., Aronoff, R., and Maquat, L.E. 2001. Identification and characterization of human orthologues to Saccharomyces cerevisiae Upf2 protein and Upf3 protein (Caenorhabditis elegans SMG-4). Mol. Cell. Biol. 21: 209-223.

Shibuya, T., Tange, T.O., Sonenberg, N., and Moore, M.J. 2004. eIF4AIII binds spliced mRNA in the exon junction complex and is essential for nonsense-mediated decay. Nat. Struct. Mol. Biol. 11: 346-351.

Unterholzner, L. and Izaurralde, E. 2004. SMG7 acts as a molecular link between mRNA surveillance and mRNA decay. Mol. Cell 16: 587-596.

Yamashita, A., Ohnishi, T., Kashima, I., Taya, Y., and Ohno, S. 2001. Human SMG-1, a novel phosphatidylinositol 3-kinase- related protein kinase, associates with components of the mRNA surveillance complex and is involved in the regulation of nonsense-mediated mRNA decay. Genes \& Dev. 15: 2215-2228. 


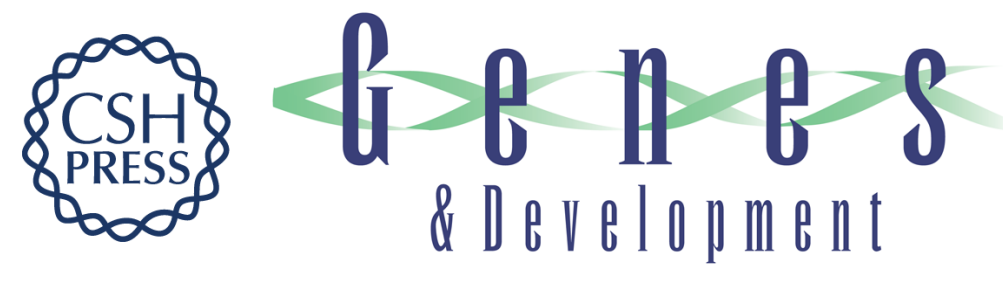

\title{
Quality control of gene expression: a stepwise assembly pathway for the surveillance complex that triggers nonsense-mediated mRNA decay
}

Isabelle Behm-Ansmant and Elisa Izaurralde

Genes Dev. 2006, 20:

Access the most recent version at doi:10.1101/gad.1407606

\begin{abstract}
Related Content Binding of a novel SMG-1Upf1eRF1eRF3 complex (SURF) to the exon junction complex triggers Upf1 phosphorylation and nonsense-mediated mRNA decay Isao Kashima, Akio Yamashita, Natsuko Izumi, et al.

Genes Dev. February , 2006 20: 355-367

References This article cites 49 articles, 22 of which can be accessed free at: http://genesdev.cshlp.org/content/20/4/391.full.html\#ref-list-1

Articles cited in:

http://genesdev.cshlp.org/content/20/4/391.full.html\#related-urls

License Email Alerting
Service $\begin{aligned} & \text { Receive free email alerts when new articles cite this article - sign up in the box at the top } \\ & \text { right corner of the article or click here. }\end{aligned}$
\end{abstract}

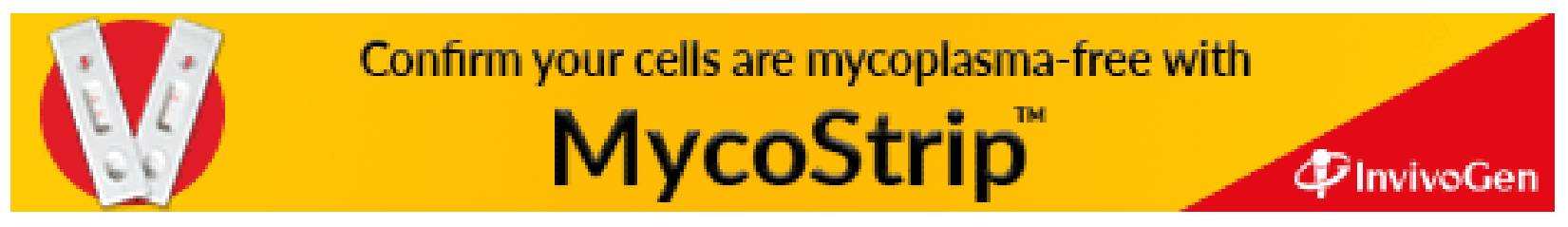

